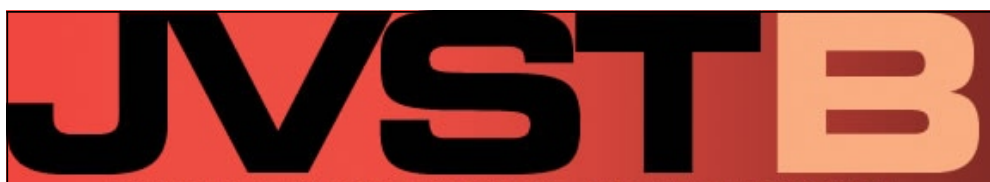

JOURNAL OF VACUUM SCIENCE AND TECHNOLOGY B

\title{
Nanostructuring GaN using microsphere lithography
}

W. N. Ng, C. H. Leung, P. T. Lai, and H. W. Choi

Citation: J. Vac. Sci. Technol. B 26, 76 (2008); doi: 10.1116/1.2819265

View online: http://dx.doi.org/10.1116/1.2819265

View Table of Contents: http://avspublications.org/resource/1/JVTBD9/v26/i1

Published by the AVS: Science \& Technology of Materials, Interfaces, and Processing

\section{Related Articles}

Improved imaging properties of thin attenuated phase shift masks for extreme ultraviolet lithography J. Vac. Sci. Technol. B 31, 021606 (2013)

Fabrication of multiscale electrodes on organic photovoltaic thin films and in situ electrical characterization by nanostencil combined with Qplus AFM

J. Vac. Sci. Technol. B 31, 021803 (2013)

Origin of defects on targets used to make extreme ultraviolet mask blanks

J. Vac. Sci. Technol. A 31, 021403 (2013)

Benefits of plasma treatments on critical dimension control and line width roughness transfer during gate patterning

J. Vac. Sci. Technol. B 31, 012205 (2013)

Design and fabrication of a metallic nanostamp using UV nanoimprinting and electroforming for replicating discrete track media with feature size of $35 \mathrm{~nm}$

J. Vac. Sci. Technol. B 31, 011801 (2013)

\section{Additional information on J. Vac. Sci. Technol. B}

Journal Homepage: http://avspublications.org/jvstb

Journal Information: http://avspublications.org/jvstb/about/about_the_journal

Top downloads: http://avspublications.org/jvstb/top_20_most_downloaded

Information for Authors: http://avspublications.org/jvstb/authors/information_for_contributors

\section{ADVERTISEMENT}

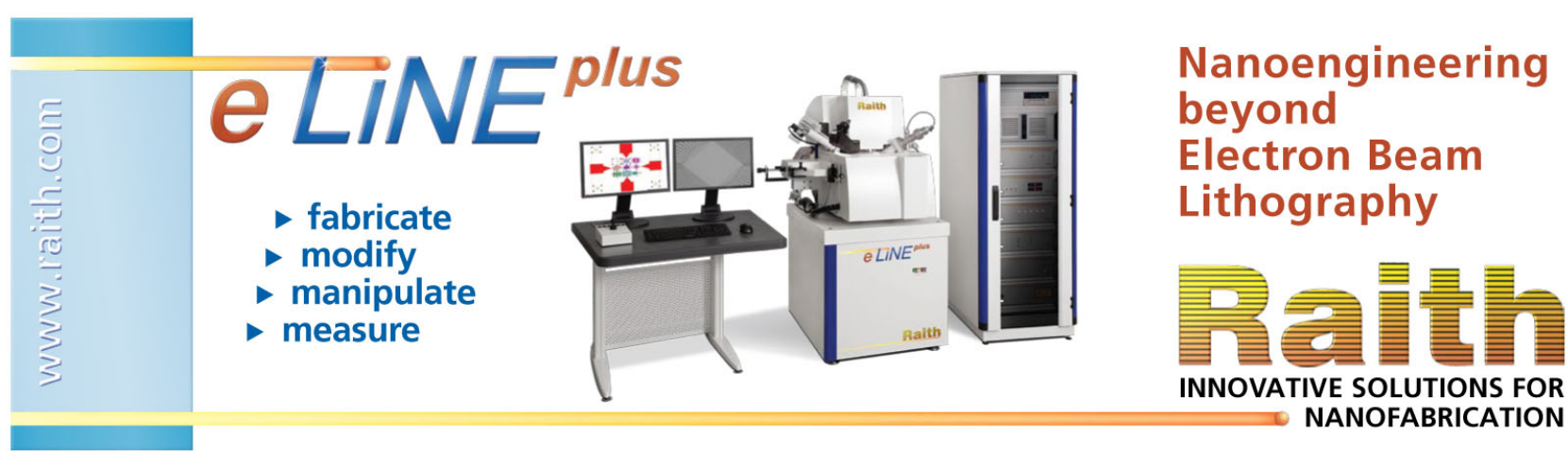




\title{
Nanostructuring GaN using microsphere lithography
}

\author{
W. N. Ng, C. H. Leung, P. T. Lai, and H. W. Choia) \\ Department of Electrical and Electronic Engineering, University of Hong Kong, Pokfulam Road, Hong \\ Kong, China
}

(Received 3 August 2007; accepted 6 November 2007; published 4 January 2008)

\begin{abstract}
The authors report on the fabrication and characterization of nanopillar arrays on GaN substrates using the technique of microsphere lithography. Self-assembled hexagonally packed silica microsphere arrays were formed on $\mathrm{GaN}$ wafers by spin coating and tilting. By precision control of process parameters, a monolayer can be formed over a wide region. The silica microspheres act as a hard mask for pattern transfer of the nanostructures. After dry etching, arrays of nanopillars were formed on the surface of the wafer. The ordered nanostructures can be clearly seen in the scanning electron microscopy images, while photoluminescence measurements revealed a twofold enhancement of light emission intensity. (c) 2008 American Vacuum Society.
\end{abstract}

[DOI: $10.1116 / 1.2819265$ ]

\section{INTRODUCTION}

Dimensional down-scaling is an inevitable trend in GaNbased materials and devices just like its Si counterparts. In order to enhance device performance, micro- and nanoscale features, in various geometries of disks, rings, and hexagons, have been incorporated into GaN light emitting diodes (LEDs). ${ }^{1,2}$ Such structures have been demonstrated to improve efficiency and directionality of the photon extraction from the mesa by overcoming total internal reflections. ${ }^{3}$ Nanoscale features, including photonic crystals, offer superior optical properties to devices by exploiting the photonic band gap. ${ }^{4}$ While microscale patterns can be readily achieved by conventional photolithography, electron-beam lithography is the most widely used method for nanopatterning for optoelectronic devices. Nevertheless, the process of $e$-beam writing is expensive and inefficient, making it unsuitable for mass production. On the other hand, the resolution of DUV lithography is limited by diffraction effects, despite its higher throughput. This encourages the development of alternative methods for nanostructure patterning on a production scale. Microsphere lithography is a potential candidate for this purpose. Randomly dispersed or ordered arrays of microsphere particles can be used as a photolithographic mask, dependent on the application involved. For example, the formation of photonic crystals would require a high degree of ordering. Being substrate independent, microsphere lithography can be applied to most material systems.

In this work we demonstrate the fabrication of ordered array of nanopillars, based on this controllable and economical technique of microsphere lithography. Originally developed by Deckman et al. to define a large area lithographic mask using a self-assembled spherical colloidal mask, ${ }^{5}$ it was further optimized by Hultenn et al. for applications involving surface-enhanced Raman spectroscopy. ${ }^{6,7}$ We adopt a similar

\footnotetext{
a) Author to whom correspondence should be addressed. Electronic mail:
} hwchoi@eee.hku.hk but modified approach to integrate ordered nanostructure arrays into GaN materials for the enhancement of optoelectronic device performance.

\section{EXPERIMENTAL DETAILS}

Schematic diagrams illustrating the fabrication process flow are shown in Figs. 1(a)-1(c). The starting material in this work is a GaN-based LED structure containing InGaN/ GaN multi-quantum wells (MQWs) [Fig. 1(a)]. Details of the device structure can be found in Ref. 8. Silica $\left(\mathrm{SiO}_{2}\right)$ microspheres from Duke Scientific, having mean diameters of 500 $\mathrm{nm}$ with a uniformity of better than $1 \%$, were used to form the lithographic mask. Silica microspheres were chosen, as opposed to polymer ones, due to their higher etch resistance, providing greater etch selectivities to $\mathrm{GaN}$. The microspheres as received from the manufacturer are suspended in deionized water and further diluted in a solution of the surfactant $n$-dodecyl-sodium-sulfate at a volume ratio of 5:1. The function of the surfactant is to lower the surface tension of the surface and thus assist the beads in spreading across the wafer surface. Monolayer formation is critical for subsequent pattern transfer; this is achieved by maintaining stringent process parameters which include concentration of microsphere suspension, dispense volume, and dimensions of the sample. A predetermined amount of the solution is precisely dispensed using a Capp mechanical micropipette with $1 \%$ precision onto a wafer-diced $10 \times 10 \mathrm{~mm}^{2}$ sample. Using a combination of tilting and spin-coating, the microspheres are dispersed and self-assemble into ordered hexagonal monolayer arrays as the liquid evaporates [Fig. 1(a)]. Both the tilt angle and rotation speeds are maintained to ensure coating uniformity across the sample and between samples. Selective coating is also possible with the introduction of a patterned photoresist masking layer. The coated sample was subsequently dry etched by inductively coupled plasma (ICP) etching using $\mathrm{Cl}_{2}$ and $\mathrm{BCl}_{3}$ as process gases at flow rates of 20 and $10 \mathrm{sccm}$, respectively, to remove the GaN materials in the void regions between the spheres for durations ranging from 30 to $120 \mathrm{~s}$. The ICP and platen powers were main- 

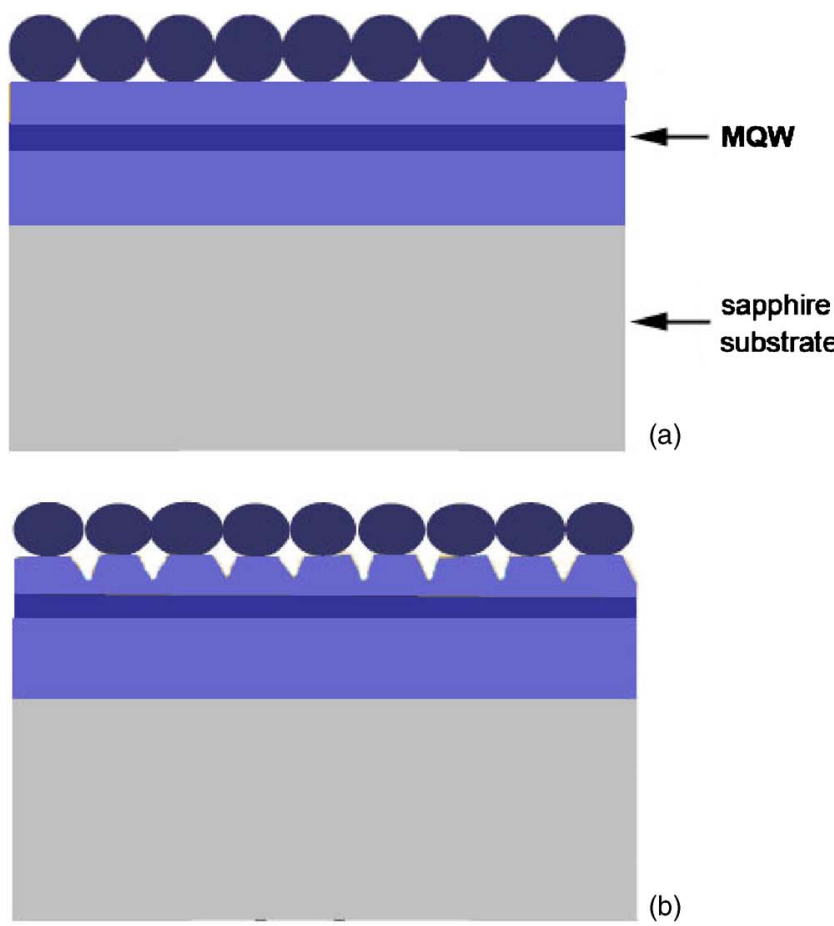

(b)

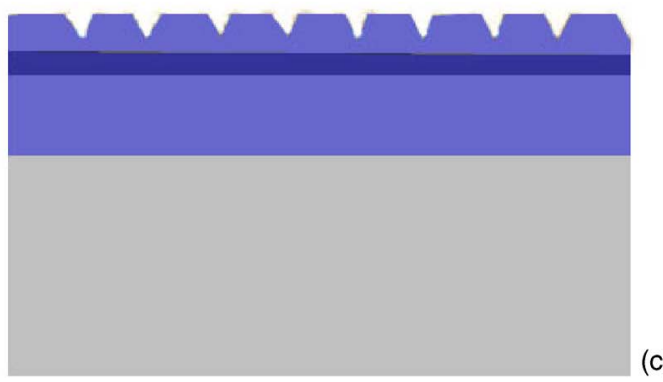

FIG. 1. Schematic diagrams demonstrating the process flow of microsphere lithography. Regularly spaced silica microspheres are coated conformally onto a GaN LED wafer in (a). During dry etching, the void regions between the tightly packed spheres are exposed to plasma and etched away, leaving behind air holes as illustrated in (b). After microsphere removal, a nanopillar array is formed as shown in (c).

tained at 500 and $100 \mathrm{~W}$, respectively, while the chamber pressure was held at $5 \mathrm{mTorr}$. Such process conditions produce an etch rate of $250 \mathrm{~nm} / \mathrm{min}$ and an etch selectivity of 4:1 between $\mathrm{GaN}$ and $\mathrm{SiO}_{2}$. The same method can be employed for the fabrication of nanopores or nanopillars; this is dependent on the etch anisotropy and duration. The ordered nanopillars after the ICP dry etch process, prior to microsphere removal, are shown in Fig. 1(b). The sample was then dipped in DI water with sonification for 2 min for detachment of microspheres, giving the resultant nanopillars on the GaN surface as shown in Fig. 1(c). Trace amounts of residual silica from the microspheres may remain on the top surface of the pillars; this is readily cleaned up by dipping the sample into a dilute $\mathrm{HF}$ solution. A second dip in an $\mathrm{HCl}$ solution removes the etch-induced damage on the sidewalls of the nanopillars and completes the fabrication process.

The surface morphology of the fabricated nanopillar structures was imaged by field emission scanning electron

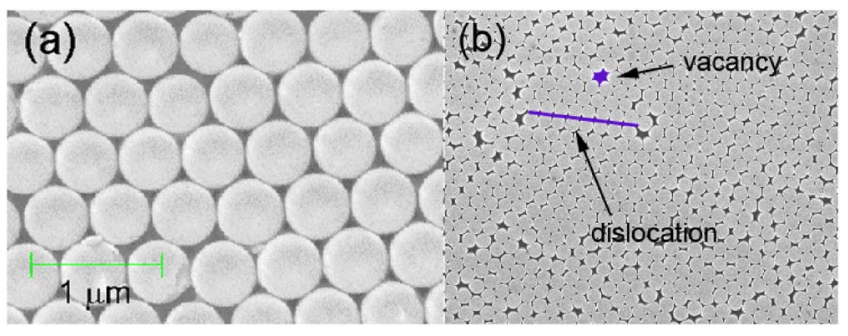

FIG. 2. (a) Silica microsphere dispensed onto GaN samples, self-assembling into ordered hexagonal monolayer arrays. (b) Various defects on microsphere coating.

microscopy (FE-SEM). Room-temperature photoluminescence (PL) spectra were collected to evaluate the optical properties of the fabricated structure, using a Spectra-Physics DPSS UV laser at $349 \mathrm{~nm}$ as an excitation source. The laser beam was focused to a spot of about $100 \mu \mathrm{m}$, and the PL signal was collected and coupled to a spectrometer via an optical fiber.

\section{RESULTS AND DISCUSSIONS}

The FE-SEM image in Fig. 2(a) shows a monolayered array of silica microspheres, self-assembled into a hexagonal fashion. The formation of the ordered array relies on the intrinsic property of the microspheres to freely diffuse across the substrate and settle down in their lowest energy configuration. The deposition of microspheres onto a substrate is a nonequilibrium process; the system tries to restore equilibrium by forming aggregates. This coating process is similar to that of atomic deposition during crystal growth. ${ }^{9}$ The microspheres migrate across the surface, which subsequently form clusters or islands by attaching to adjacent microspheres, which is dependent on the diffusion rate and concentration of microspheres. In order to form well-ordered monolayers, microspheres must adsorb on the edge of aggregates and continue to migrate around the perimeter. Thus the diffusion rate must be precisely controlled, which is achieved by optimizing the spin speed and controlling the tilt angle. Using this hybrid method, uniformly dispersed monolayer coatings span across $70 \%$ of the entire sample area, with an edge bead developed at the periphery of the samples. In this work, the microspheres are coated onto a photoresistpatterned sample, with multiple $400 \mu \mathrm{m}$ by $200 \mu \mathrm{m}$ exposed windows (which subsequently act as the emission regions of electroluminescent LEDs). Nevertheless, point defects such as vacancies or line defects such as dislocation, analogous to those formed during crystal growth, can be distinctively observed at various random locations as illustrated in Fig. 2(b). While this is sufficient as a proof-of-concept demonstrator, a reduction in defect density must be achieved for optimal optical properties.

The ordered nanopillar array was transferred onto the GaN substrate by ICP etching through the microsphere template. The void regions between microspheres permit penetration of reactive ions in the plasma and thus the removal of $\mathrm{GaN}$ materials forming air holes. Intuitively, a certain de- 


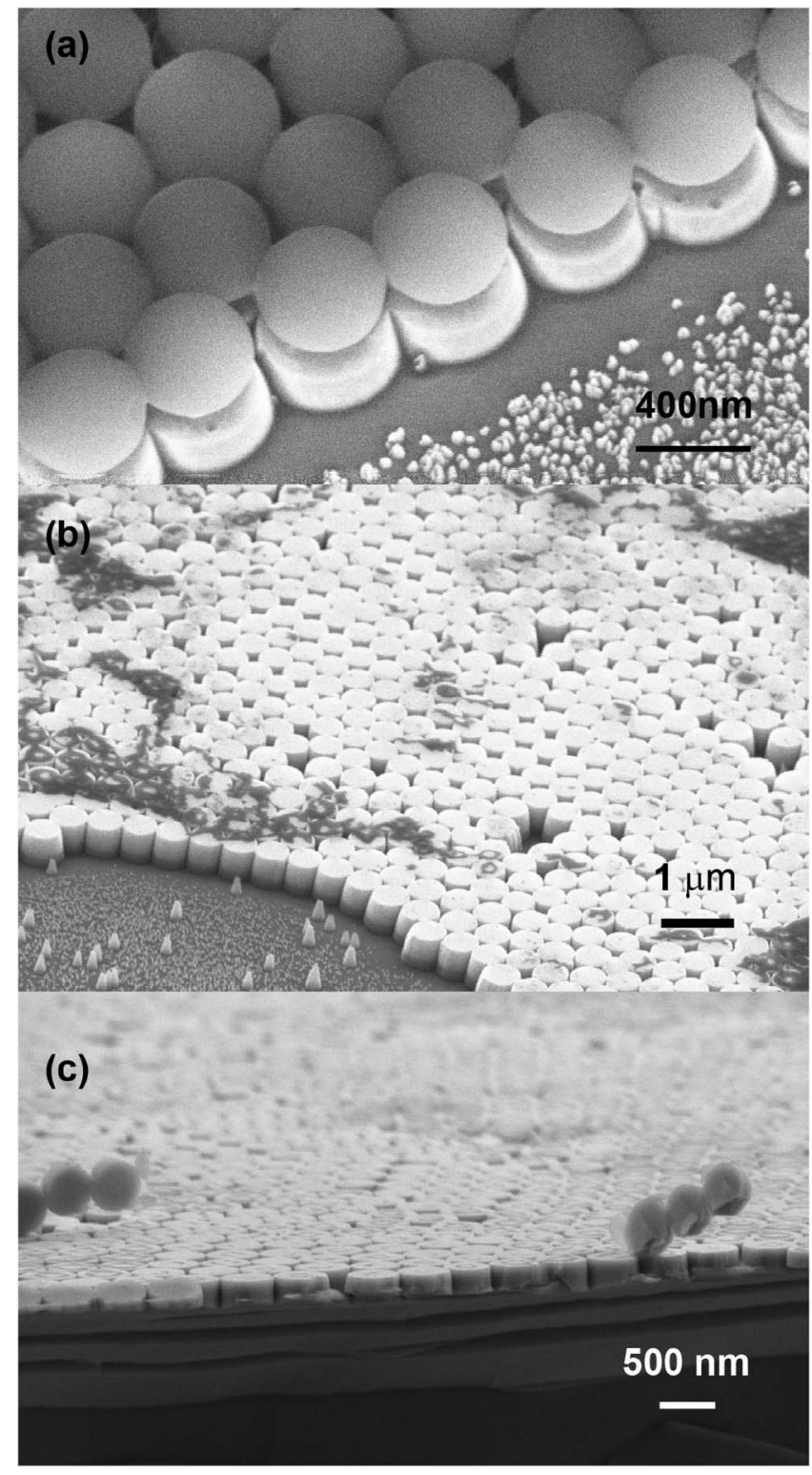

FIG. 3. (a) FE-SEM image showing microspheres sitting on top of the nanopillars after dry etching. (b) The nanopillars after microsphere removal. (c) FE-SEM image showing the cross-sectional view of nanopillars.

gree of dimensional tuning can be achieved by adjusting the reactivity and directionality of the ions (by adjusting the process parameters). By applying higher ICP powers and lower platen powers, the features are etched isotropically. As a result, the sides of the microspheres are also etched (albeit at a slower rate compared to the top), causing lateral dimension shrinking, facilitating penetration of reactive ions between the microspheres. Such process conditions ensure nanopillar formation, rather than merely creating an ordered array of air holes. A FE-SEM image showing a nanopillar array etched for a duration of $30 \mathrm{~s}$ prior to microsphere removal is shown in Fig. 3(a). At this stage the microspheres have become oval in shape as the spheres are etched faster at the top. After sonification, the microspheres are fully detached, leaving be-

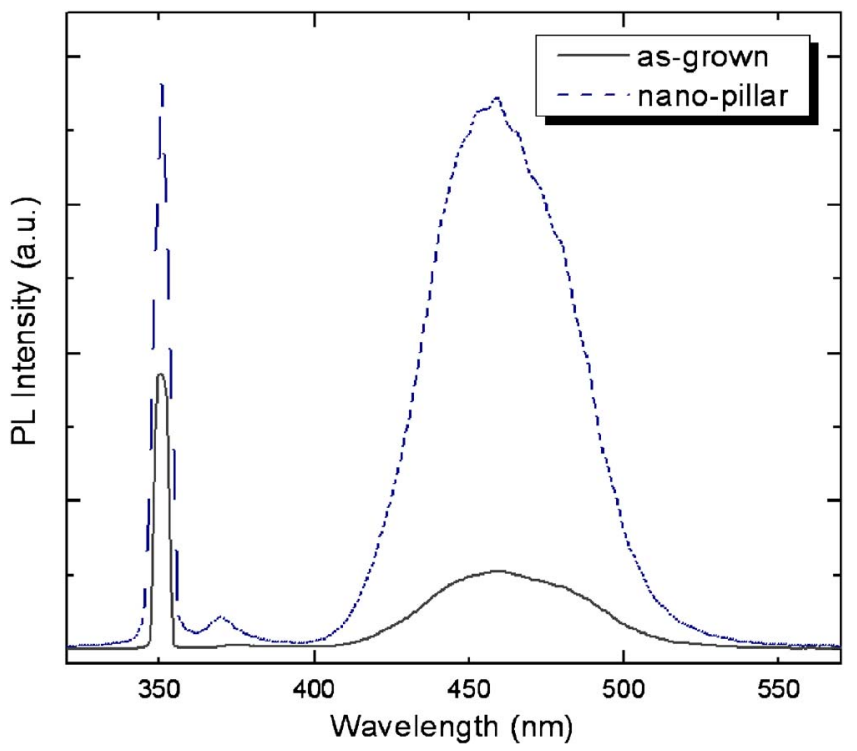

FIG. 4. PL spectra of as-grown and processed sample with nanopillar array.

hind an array of nanostructures. In Fig. 3(b), the edge region of a nanopillar array that has been etched for $60 \mathrm{~s}$ is illustrated. While the height of the pillars at the edge is approximately $500 \mathrm{~nm}$, the actual depth of the triangular holes between the pillars is expected to be shallower, as only a fraction of the incident ions can penetrate the holes. The sample was cleaved across the nanostructured region to expose the cross-section, which is shown in Fig. 3(c). From the cross-sectional view, the depth of the feature is observed to be $\sim 250 \mathrm{~nm}$, confirming that the MQWs on the nanopillars have been isolated.

To evaluate the effects of nanopillar incorporation, PL spectra were collected from the processed and unprocessed regions of the same sample, which are plotted in the graph in Fig. 4. The spectral peak centered at $460 \mathrm{~nm}$ corresponds to light emission from the InGaN/GaN MQWs in the LED structure. As evident from the plot, a twofold increase in emission intensity was observed, attributed to enhanced light extraction via the nanoscale air holes. In smooth and flat as-grown wafers, a large proportion of light is trapped in the wafer due to total internal reflections. ${ }^{8}$ Roughening of the surface, either on the micro- or nanoscale, significantly improves light extraction by allowing more photons to pass through the interface. ${ }^{10} \mathrm{~A}$ slight blueshift of about $3 \mathrm{~nm}$ was also observed, which may be attributed to partial strain relaxation, similar to the observation in microscale disk structures. ${ }^{11}$

While the fabrication of nanopillars is demonstrated in this report, the versatility of microsphere lithography can be extended to various other applications, including photonic crystals (by exploiting the regular array of air holes) and the fabrication of nanotips (which we have successfully demonstrated but will report on separately). The dimensions of the arrays may also be scaled up readily. 


\section{SUMMARY}

In summary, we have demonstrated the fabrication of nanopillar arrays on $\mathrm{GaN}$ material using the technique of microsphere lithography. A self-assembled, hexagonally closed, packed silica microsphere monolayer array was coated by spin coating and tilting, acting as a hard mask to form nanopillars with dimensions of $\sim 500 \mathrm{~nm}$ by ICP etching with various heights. The ordered nanostructures span across regions of approximately $400 \mu \mathrm{m}$ by $200 \mu \mathrm{m}$ in this work. PL measurements revealed that the presence of nanopillars enhanced the light emission intensity by about $230 \%$ due to increase in light extraction efficiency. This result can be exploited to enhance optical performance in nitride-based light emitting diodes.

\section{ACKNOWLEDGMENTS}

This work was jointly supported by a CERG grant of the Research Grant Council of Hong Kong (Project No. HKU
7121/06E) and the University Development Fund of the University of Hong Kong (Nanotechnology Research Institute, Project No. 00600009).

${ }^{1}$ H. W. Choi, C. W. Jeon, and M. D. Dawson, IEEE Photon. Technol. Lett. 15, 510 (2003).

${ }^{2}$ H. W. Choi et al., Appl. Phys. Lett. 86, 021101 (2005).

${ }^{3}$ H. W. Choi, M. D. Dawson, P. R. Edwards, and R. W. Martin, Appl. Phys. Lett. 83, 4483 (2003).

${ }^{4}$ E. Yablonovitch, Sci. Am. 285, 47 (2001).

${ }^{5}$ H. W. Deckman and J. H. Dunsmuir, Appl. Phys. Lett. 41, 377 (1982).

${ }^{6}$ J. C. Hulteen and R. P. Van Duyne, J. Vac. Sci. Technol. A 13, 1553 (1995).

${ }^{7}$ C. L. Haynes and R. P. Van Duyne, J. Phys. Chem. B 107, 7426 (2003).

${ }^{8}$ H. W. Choi, C. W. Jeon, M. D. Dawson, P. R. Edwards, R. W. Martin, and S. Tripathy, J. Appl. Phys. 93, 5978 (2003).

${ }^{9}$ H. Röder, E. Hahn, H. Brune, J. P. Bucher, and K. Kern, Nature (London) 366, 141 (1993).

${ }^{10}$ D. W. Kim, H. Y. Lee, M. C. Yoo, and G. Y. Yeom, Appl. Phys. Lett. 86, 052108 (2005)

${ }^{11}$ S. X. Jin, J. Li, J. Y. Lin, and H. X. Jiang, Appl. Phys. Lett. 77, 3236 (2000). 